\title{
POLÍTICAS PÚBLICAS PARA EL DESARROLLO RURAL: UN ANÁLISIS MULTIESCALAR
}

\author{
Mercedes Molina Ibáñez y Massimiliano Farris \\ Dpto. de Geografía Humana, Universidad Complutense de Madrid \\ moyba@ghis.ucmes; mass.farris@gmail.com
}

Resumen: El artículo reflexiona sobre el papel de las políticas públicas y de la sociedad local como protagonistas de un cambio necesario para el desarrollo de los territorios rurales marginales, desde una perspectiva multiescalar que considere la dimensión territorial. Nueva economía, multifuncionalidad rural, territorio y políticas públicas constituyen la base de este análisis.

Palabras clave: Políticas públicas, desarrollo rural, enfoque multiescalar, territorialidad.

\begin{abstract}
This paper analyses the role of the public policies and of the local society as protagonists of a change necessary for the development of the rural marginal territories at different scales. New economy, rural multifunctionality, territory and public policies constitute the base of this analysis.
\end{abstract}

Keywords: Public policies, rural development, multiple-scale approach, territoriality.

Recibido: 9-12-10. Aceptado: 22-11-11. 


\section{Competitividad territorial y políticas públicas de desarrollo rural, según una visión multiescalar}

La globalización económica no tiene una proyección territorial homogénea, la tipología de espacios que configura es muy diversa y en ella el peso del territorio tiene una especial significación a la hora de impulsar procesos y determinar actividades (Santos, 1994). La actividad económica de mayor valor añadido se está concentrando en las regiones "centrales" y en muy pocas áreas periféricas que han encontrado su nicho de mercado en la economía global, quedando en estado precario otras, muy numerosas en el mundo, que se identifican sobre todo con las rurales. Incluso dentro de los territorios protagonistas, se aprecian importantes desigualdades internas derivadas, por una parte del modelo de concentración territorial dominante -caso de la metropolización (Davis, 1992; Merrifield y Swyngedouw, 1997)- de la exclusión social -como es el fenómeno del "sin hogarismo" (Bachiller, 2009 para el caso madrileño) - y de las necesidades de trabajos no cualificados o marginales asociados a numerosas actividades globales y en parte resueltas por la inmigración (Sassen, 2005).

Aunque a grandes rasgos se puede decir que un resultado territorial del capitalismo globalizado es la urbanización del espacio y la constitución de metrópolis globales, la causa de esta relación privilegiada no depende exclusivamente de la concentración de la "economía informacional" (Castells, 1997) y de los flujos financieros en el espacio urbano. Hay múltiples factores, además de los meramente económicos, que concurren en la consolidación de las ventajas que hacen un territorio competitivo: de tipo socio-cultural (el fenómeno “buzz”, Storper y Venables 2004), político (el proceso de rescaling del Estado-nación, Brenner 2003) o bien ambiental.

Aunque todo se desenvuelva en el marco de la competitividad global y, por ello, la escala global y la metropolitana son los niveles que centralizan la atención, los procesos capitalistas territorializados no se resuelven exclusivamente en ellas. Es importante considerar, de la misma manera, las relaciones entre la escala metropolitana y la escala estatal con la regional o la local ya que pueden, en algunos casos y en términos jerárquicos, determinar los procesos globales e influirse mutuamente.

Interpretar el posicionamiento competitivo de los territorios como si fueran empresas que compiten entre si, aclara cómo, hoy en día, se instauran las relaciones entre los mismos. Para tener una visión más completa de estas relaciones, a la hora de implementar políticas de desarrollo, es preciso leer la competitividad territorial en términos multiescalares, haciendo referencia a las investigaciones sobre las escalas del capitalismo (Harvey, 2004; Swyngedow, 2000; Brenner, 1999; Howitt, 1993, entre otros) y a los estudios sobre la división del trabajo (Santos, 1996). Por las características que determinan las pautas de concentración del proceso de globalización, en el actual contexto del capitalismo, también los lugares tienen que competir para emer- 
ger, así como lo hacen las empresas, creando sus propias ventajas comparativas (Camagni, 2006). Los territorios actúan como sujetos activos que compiten para lograr sus intereses, atrayendo recursos económicos y humanos. Factores naturales, demográficos, sociales, políticos económicos y culturales desarrollados a lo largo del tiempo, pueden determinar ventajas, o desventajas, a partir de las cuales las administraciones locales deben planificar sus estrategias. Para activar procesos de desarrollo a largo plazo, los territorios, así como las empresas, tienen que trabajar del lado de la oferta, demostrando ventajas absolutas o competitivas que los posicionen en una condición preferente respecto de los demás. Dentro de la competitividad territorial, lo rural ocupa un lugar muy poco o nada destacado a efectos de atraer inversión internacional. Sin embargo, muchos acontecimientos globales sí que repercuten directa o indirectamente en el ámbito rural y de hecho muchas regiones de Europa, se ven a menudo afectadas por procesos globales que, en unos casos, pueden desencadenar importantes problemas y, en otros, determinar ciertos beneficios territoriales. Importantes aspectos de política internacional tendrán efectos sobre una parte de la actividad económica regional, sobre todo de la agraria. Es el caso de las decisiones de la Organización Mundial del Comercio (OMC), relacionadas con el aumento de la liberalización del comercio internacional, así como por los efectos de la incorporación de China en dicho organismo. Asimismo se pueden señalar los comportamientos de espacios con un cierto grado de integración, tal como se va configurando el MERCOSUR en su tendencia a constituirse como un mercado integrado sobre el modelo de la UE y por el interés de establecer con la misma un tratado bilateral de libre comercio. En el momento que estas tendencias se concreten, habrá repercusiones sobre algunas políticas sectoriales europeas que tendrán necesariamente que modificar su estrategia. Acontecimientos internacionales y globales pueden incidir en la reflexión sobre el contenido de algunas políticas públicas, tradicionalmente orientadas hacia el proteccionismo agrario y por ello con una clara dimensión sectorial, y justifican un cambio de enfoque en esas políticas, vinculándolas más a la dimensión territorial.

A la vez, otros aspectos relacionados con lo global, oportunamente fomentados y acompañados por políticas públicas adecuadas, pueden tener efectos positivos sobre estos territorios rurales (Marsden et al. 1993). En principio cabría considerar las nuevas concepciones del desarrollo (Bardají, 2008; Molina, 2004a; Moyano y Paniagua, 1996), cada vez más reclamadas por amplios sectores sociales que hoy adquieren gran valor ante la crisis del modelo productivo dominante y que van unidas a la consecución de una globalización más humanizada, orientada hacia un mayor equilibrio socio-territorial, la defensa cada vez más comprometida del desarrollo sostenible y la superación de concepciones económicas orientadas hacia el simple crecimiento. Es preciso insistir sobre el desarrollo humano, la calidad de vida y otros valores ignorados hasta hace poco. Ello obedece a importantes cambios en la sociedad que, a la 
vez, provocan modificaciones de ciertos comportamientos sociales; entre desarrollo y sociedad se está produciendo una relación directa de causa-efecto, de gran trascendencia. Su expresión más inmediata se traduce, al menos en sociedades desarrolladas, en el paso, aunque todavía muy lento, de una producción orientada hacia la cantidad a la búsqueda de la calidad, diferenciación y seguridad de determinados productos para sectores específicos del mercado. Todo ello puede contribuir a la puesta en valor de nuevos y tradicionales recursos, destacando los naturales que en parte se localizan en regiones históricamente menos dinámicas y que constituyen el motor de los nuevos desarrollos (Villarino, 2000; Sassu, 2004; Molina, 2004b; Perelli et al, 2010). Asimismo puede permitir la diversificación de las economías locales en actividades multifuncionales que van a reclamar, para que sea efectiva su transformación, nuevas dinámicas sociales, de ordenación y conexión territorial así como nuevos compromisos políticos. Existen posibilidades de cambio porque se consolida un mercado que reclama nuevos productos y es muy importante, en el momento actual, aprovechar esta situación para ir creando paulatinamente un territorio competitivo por sus características, por su economía y por su sociedad. Es decir, un territorio que sea capaz, en un futuro, de afrontar cualquier decisión política de cambio internacional o de la propia UE. Todo ello, hace que un territorio en su conjunto, y no sólo una actividad, se singularice en el nuevo modelo económico y adquiera una dimensión propia, como es el caso de los territorios rurales.

Global y rural no son procesos enfrentados, ni metropolitano y rural son territorios excluyentes, por el contrario entendemos que cada vez tendrán una mayor conexión; la dificultad radica en el "cómo" y a partir del "qué". En este sentido cabe señalar que diferentes comportamientos a nivel global pueden incidir en los actuales contenidos de las políticas públicas aplicables a estos territorios. En el ámbito de la Unión Europea, se deberán impulsar políticas territoriales encaminadas a subsanar los estrangulamientos que no corrige el mercado y, así, poder gestionar de forma innovadora y sostenible los recursos locales y facilitar un nuevo encuentro entre oferta local y las exigencias actuales de una demanda que procede mayoritariamente de la sociedad urbana y metropolitana.

\section{Territorialidad trans-escalar: interpretar los efectos territoriales de las políticas públicas para el desarrollo rural}

Para implementar procesos de desarrollo, el análisis de la acción e intención de los sujetos sociales y políticos debe tener presente la fuerza que poseen las adminis- 
traciones públicas en el gobierno del territorio, tanto en la escala del Estado-nación como de las instituciones locales. La noción de territorialidad permite aclarar las relaciones entre modalidades de la acción colectiva y ámbitos territoriales en los cuales se desarrolla, según un enfoque multiescalar. Es decir, la territorialidad reúne, en si misma, los vínculos funcionales entre tres elementos del desarrollo local: territorio, práctica social y políticas públicas.

Tal como plantea Pasqui (2005) que el desarrollo siempre es territorial, en el sentido de que las prácticas que lo connotan tienen una relación con una especificidad territorial, que además contribuyen a reproducir. Esto implica que los procesos de transformación territorial y de desarrollo que persiguen conectar la escala local con la global, necesitan tener como "ingredientes" básicos las especificidades territoriales y los actores locales, valorizando así las potencialidades endógenas de cada lugar y la construcción de las identidades colectivas de los sujetos locales (Governa, 2005). Para ello, se debe superar la visión que interpreta el territorio como un mero soporte sobre el cual aplicar de forma exógena intervenciones estandardizadas y compartimentadas de tipo infraestructural, industrial, turístico o social. Es necesario abandonar el enfoque "de arriba-abajo" que ha caracterizado muchas políticas públicas, para pasar a una "desde abajo" o "de abajo hacia arriba". Esto implica una visión multidimensional, integrada e intersectorial de las políticas (Governa, 2005) y la negociación formal entre los actores y los intereses. Con la superación de la visión "de arriba-abajo" de las políticas, sí se da un cambio en la concepción del territorio: éste se considera como un patrimonio común que es necesario valorizar (Magnaghi, 2000), es el punto de referencia a partir del cual y en el cual construir y evaluar políticas y acciones.

En la reinterpretación del valor del territorio, de cara a los procesos de desarrollo local, la territorialidad asume, a nuestro juicio, un valor central. Una superación de la visión weberiana del Estado y de la territorialidad se ha producido con las investigaciones sobre las mutaciones en la naturaleza del Estado-nación que han surgido en relación con el proceso de globalización del capitalismo (Sassen, 1991; Swyngedouw, 2000; Brenner, 2003). Ahora bien, como señala Governa (2005) es más correcto hablar de "territorialidades", cuya dimensión es trans-escalar. Los cambios que se están produciendo conllevan una redefinición, tanto por las "Sociedades" como por las "Comunidades" locales, de los conceptos de pertenencia, identidad y de la acción colectiva en base territorial (Farinós, 2005). Estos pueden adquirir, contemporáneamente, un valor diferente en las diversas escalas, sin que ello implique una contradicción. Un colectivo social se puede identificar con su pertenencia a la escala territorial de su ciudad o de su región o de su Estado en forma diferente en relación a la acción que quiere desarrollar en cada una de estas diferentes escalas. De este modo, se redefine también la construcción de los territorios y de la territorialidad que se caracteriza como trans-escalar. La transcalaridad de ésta, está determinada por la intersección entre la multiplicidad de escalas en las cuales un territorio es llamado a posi- 
cionarse y la asunción sincrónica de varios y diferentes roles sociales, determinados por la variedad de relaciones de poder que los individuos viven e interpretan como actores políticos, económicos y sociales.

La transcalaridad de la territorialidad es un elemento clave para poder interpretar las conexiones entre global y local. Para leer estas conexiones en perspectiva de desarrollo, es necesario referirse también a la otra dimensión de la territorialidad, la práctica social, es decir cómo una sociedad local gestiona sus dinámicas internas en relación a su entorno y a los condicionantes exógenos.

Los comportamientos de los actores sociales y las relaciones entre las formas del gobierno territorial con las diferentes escalas, pueden distinguirse en dos tipos de territorialidad: una "pasiva" y otra "activa" (Governa, 2005). En el primero, son los sujetos que detentan y ejercen el poder (económico o administrativo) quienes ponen en marcha acciones activas e innovadoras, a menudo persiguiendo estrategias de control que tienden a la exclusión social y a la gestión elitista de los recursos. El otro resulta de una práctica social colectiva y compartida de los sujetos locales. Interpretada en una proyección trans-escalar, el análisis de la práctica social induce a acercarse al problema del reparto de competencias entre el Estado y las entidades locales (Dematteis y Governa, 2005). En este caso, una "territorialidad activa", se desprende de estrategias de inclusión orientadas a valorar las especificidades locales en base a la confrontación de las necesidades de todos los sujetos implicados. Estas estrategias son consecuentes con la implementación de políticas "de abajo hacia arriba". Éstas producen un progresivo alejamiento del centro de gravedad de la toma de decisiones hacia los niveles inferiores, con la consecuente acogida de las voluntades locales en las escalas supra-locales. De esta manera se pueden desactivar las tensiones individualistas y separatistas. Una territorialidad así concebida está relacionada con la noción de "gobernanza". La primera se puede interpretar como la aplicación, a la dimensión territorial, de la segunda: es decir una coordinación de la participación de una multiplicidad de actores, que se desempeñan en diferentes escalas y en procesos económicos y sociales. Para que se verifiquen las condiciones para un desarrollo local, las políticas públicas, y también la organización del Estado, deben metabolizar, asumir como propio, el contenido de la territorialidad activa y su dimensión trans-escalar.

Implementar un proceso de "gobernanza", mediante políticas de territorialidad activa, implica, no sólo descentralizar el proceso de toma de decisiones de la escala estatal a las locales, sino, a la vez, la superación del "municipalismo". Por esto, consideramos importante instrumentos político-administrativos supramunicipales, expresión de la territorialidad "local y rural" que pueden asumir las mediaciones necesarias de escala, no sólo en la fase de la aplicación de políticas de desarrollo, determinadas a niveles superiores, sino sobretodo en la fase de propuesta. La comarca, pensamos, puede constituirse como la escala del desarrollo local rural. Ella, 
en primer lugar, se podría constituir como la instancia institucional que acoja las solicitudes individuales y colectivas vinculadas al territorio que, luego, deben ser transmitidas a las escalas superiores. De esta manera se puede instaurar un flujo constante, tanto horizontal como vertical, de estrategias de desarrollo, en las cuales estén representadas las exigencias de todas las escalas.

La escala territorial del desarrollo local debe plantearse en base a una triple dimensión: administrativa (los límites territoriales de aplicación de las estrategias de desarrollo), ambiental (territorio como patrimonio) y social (territorio como "construcción social"). La primera dimensión se justifica considerando que el desarrollo local no se "mueve" con la misma "facilidad" que el capitalismo. Las dinámicas capitalistas definen las ventajas locacionales y la geografía de las relaciones sociales de producción, "construyendo" escalas que trascienden los límites administrativos y que se justifican sólo hasta que se dan las condiciones para una reproducción del capital. Por su parte, los procesos de desarrollo local están condicionados por las escalas capitalistas pero, además, por las escalas administrativas. Por ser el resultado de varios factores (territorio, territorialidad y políticas públicas) las acciones de desarrollo local tienen que volcarse en la dimensión administrativa local (municipal y supra-municipal). El objetivo es no encerrarse en unos límites administrativos sino interpretar las relaciones políticas internas y también las que se instauran "hacia afuera", en forma horizontal (entre mismas escalas, por ejemplo municipios) o vertical (trans-escalares). No se puede prescindir de la dimensión municipal, pero sí impulsar acciones para superar esos límites (territorios GAL, mancomunidades y sobretodo comarcas).

En relación a la segunda dimensión, resultan sugerentes las consideraciones de Magnaghi (2000) el cual propone que las relaciones de desarrollo entre la población local y su "patrimonio territorial" deben establecerse según tres factores: en primer lugar, el valor del patrimonio no se identifica primariamente con su valor de uso; luego, todo el territorio es patrimonio y tiene que ser considerado como un ecosistema de alta complejidad; finalmente, el desarrollo local no tiene fronteras, escalas, ni actores previamente constituidos. Esto no quiere decir que el proceso sea espontáneo, indudablemente se necesitan "chispas" que lo enciendan e, inevitablemente, sí serán determinados actores quienes lo generarán en una determinada escala. Es de esperar que los límites geográficos, dentro de los cuales el proceso de desarrollo puede darse, sean flexibles y con una proyección espacial y temporal, activando relaciones horizontales y verticales dentro del territorio y hacia afuera. Estas formas de aprovechamiento, o mejor dicho, de "cuidado" del territorio como "patrimonio", pueden dar una respuesta integral a los problemas de la sostenibilidad del desarrollo y, a la vez, inducen a buscar nuevas formas de participación y representatividad política. Se introduce así la tercera dimensión de la escala del desarrollo local, es decir considerar el territorio como una "construcción social" mediante procesos históricos de larga duración donde se instauran relaciones e influencias mutuas entre acción 
humana y ambiente natural. A este nivel, la sostenibilidad del desarrollo no se puede reducir exclusivamente a la esfera del medio ambiente o de la economía, debe también insertarse en la política. Por esta razón, autosostenibilidad del desarrollo y autodeterminación en el gobierno del territorio (Magnaghi 2000), se encuentran y complementan en la reunificación de la figura del "ciudadano" con la del "productor" e implica el establecimiento de nuevas formas, holísticas, de "cuidado" del territorio.

Para que existan soluciones eficaces a los problemas de los territorios rurales marginales son determinantes las políticas públicas que consideran el territorio en su conjunto y no sólo un sector económico. Es decir, políticas territoriales que sean capaces de generar competitividad, incentivar actuaciones y propiciar inversiones en zonas desfavorecidas y, en muchos casos, despobladas. Todas las actuaciones que se realicen en los territorios rurales deben estar sustentadas sobre un conocimiento, un compromiso y una participación de la sociedad local que debe alzarse en actor y director del cambio. Es fundamental para ello contribuir a cambiar actitudes y mentalidades, vencer apatías y victimismos, superar los individualismos e impulsar el cambio de: "el productor agrario tradicional al empresario rural innovador". Para ello resulta imprescindible informar y formar a la población local, establecer su participación a la hora de definir necesidades y actuaciones, así como incentivar nuevos empresarios locales, especialmente entre jóvenes y mujeres. El desarrollo rural, dada la naturaleza, la problemática y las potencialidades de sus territorios exige una estrategia múltiple que integre las políticas públicas orientadas preferentemente a garantizar la sostenibilidad de sus recursos, la creación de infraestructuras, a la formación de recursos humanos, al incentivo de la clase empresarial y a la ordenación del territorio, poniendo siempre en primer lugar la participación de la población local. En suma, Administraciones públicas cohesionadas junto con la sociedad local, constituyen el binomio imprescindible para alcanzar objetivos significativos y de impacto socioeconómico y ambas, junto con la implantación progresiva de esa producción multifuncional definida, exigen unas relaciones multiescalares.

\section{Conclusiones}

Con frecuencia asistimos al debate entre la necesidad o no de una políticas públicas entre el mayor o menor protagonismo del mercado, a la hora de abordar el crecimiento de una economía, pero, sobre todo, en el momento de definir un desarrollo territorial. La pregunta difícil de responder es que tipo de políticas se necesitan: sectoriales o territoriales, de incentivos o de subvención y, por supuesto, a qué escala, nacional, regional, local o en su caso, supranacional. Hemos insistido con frecuencia en la necesidad de contemplar cualquier proceso económico con una verdadera dimensión territorial, tanto en la concerniente a escala global como local, dada la 
necesidad de comprender y de interpretar el territorio en su relación causa-efecto con los diferentes procesos. Por ello entendemos que las políticas públicas que afectan al mundo rural, deberían considerar en mayor medida la dimensión territorial frente a la sectorial. El desarrollo rural debe integrar todo, lo agrario, lo forestal, lo industrial y los servicios, definiéndose la multifuncionalidad por las especialidades y recursos territoriales y no sólo por la actividad agraria. Sería muy concerniente abordar en este sentido las complementariedades económicas que se pueden y deben establecer en el rural. Por otra parte, entendemos que las políticas públicas debería estar articuladas entre diferentes escalas y, si bien son necesarias aquellas supranacionales, caso de la Unión Europea, nunca deberían perder la perspectiva local y el diseño desde abajo, para lo cual la flexibilidad en su aplicación debería ser prioritaria. Hay que tener siempre presente que la heterogeneidad es el rasgo dominante de los territorios rurales y ello introduce una importante dificultad a la hora de aunar intereses e integrar actuaciones, máxime cuando en numerosos países caso por ejemplo del español, no se ha superado el municipalismo.

Asimismo es importante, si se considera esa visión integrada de lo rural, evitar la dispersión de numerosas políticas públicas. Si consideramos aquellas propias del Estado o de Diferentes Comunidades Autónomas, las políticas de infraestructuras siguen unos objetivos, las del sector agrario y forestal otros, las de turismo otros más y lo mismo se podría decir de las de patrimonio o de medioambiente, para cerrar el círculo. Este hecho impide, en ocasiones, actuar con eficacia y proyectar las ventajas de una política en otro sector, la coordinación es imprescindible.

Por ello, cuando se cuestionan las políticas públicas en general, deberíamos analizar en profundidad sus problemas, sus fallos y sus repercusiones y a partir de una investigación rigurosa interrelacionar aspectos que pueden determinar crecimientos económicos y desarrollos territoriales equilibrados y eficaces. La crisis actual no sólo ha puesto de manifiesto la vulnerabilidad de los mercados, sino también la debilidad de algunas políticas o su ausencia en sectores clave. Probablemente, junto a las reflexiones que nos pueden determinar ciertos modelos económicos sustentados sobre actividades que han resultado muy vulnerables, habrá que añadir la importancia que deben tener los Estados o los gobiernos en diferentes escalas territoriales y determinar nuevas formas de actuación en el diseño de sus propias políticas. Mercado y Estado deben seguir conviviendo y este último no deberá tener otros protagonismos, tales como controlar los excesos del primero, velar por una justicia social y actuar considerando las bases que pueden y deben determinar innovaciones futuras, sobre todo a partir de la educación y la investigación, pero también deberá contemplar la importancia de la solidaridad territorial, casi siempre ignorada. En esta última consideración el diseño de unas políticas públicas aplicadas a lo rural debería ser prioritario, a efectos de generar territorios más competitivos como base para impulsar nuevos y tradicionales recursos. Si tratamos de establecer una estrecha relación entre 
I+D+I, quizás sería interesante considerar Investigaciones + Políticas Públicas + Desarrollo (I+PP+D), en su aplicación rural, con una orientación territorial.

El debate quizás no debería estar entre políticas sí o no, más bien deberíamos considerar qué políticas necesitan determinados territorios para afrontar los retos del siglo XXI.

\section{Bibliografía}

Bachiller, S. (2008). Exclusión Social, Desafiliación y Usos del Espacio. Una etnografia con personas sin hogar en Madrid. Tesis doctoral por la UAM; con fecha de defensa 27 de marzo de 2008

Bardají, I, Ramos F. Ramos, E. (2008). "Los nuevos espacios rurales" en Papeles de Economía Española, nำ 117: 191-207.

Boisier, S. (2001). "Desarrollo (local). ¿de qué estamos hablando?" en Madoery, O y Vázquez Barquero, A. (eds.), Transformaciones globales, Instituciones y Politicas de desarrollo local. Editorial Homo Sapiens, Rosario, 2001.

Brenner, N. (2003). "La formación de la ciudad global y el re-escalamiento del espacio del Estado en la Europa Occidental post-fordista" en EURE (Santiago), vol 29, n. 86. Santiago de Chile.

Brenner, N. (1999). "Globalisation as Reterritorialisation: The Re-scaling of Urban Governance in the European Union" en Urban Studies Vol. 36, N. 3: 431-451.

Camagni, R. (2006). "Acerca de la solidez del concepto de competitividad territorial", en Torroja, A y Camagni, R. Una nueva cultura del territorio. Criterios sociales y ambientales en las políticas y el gobierno del territorio. CUIMPB, Barcelona.

Castells, M. (1997). La era de la información. Economía, sociedad y cultura. Alianza Editorial, Madrid.
Davis, M. (1992). City of quartz. Excavating the future in Los Angeles. Vintage Books, New York.

Dematteis, G. y Governa, F. (2005). "Il territorio nello sviluppo locale. Il contributo del modello SloT" en Dematteis, G. y Governa, F (eds) Territorialitá, sviluppo locale, sostenibilitá: il modello SLoT, Franco Angeli, Milano.

Farinós Dasí, J. (2005). "Nuevas formas de gobernanza para el desarrollo sostenible del espacio relacional" en Ería, 67 págs 219-235.

Governa, F. (2005). "Sul ruolo attivo della territorialità" en DE MATTEIS, G. y GOVERNA, F (eds) Territorialitá, sviluppo locale, sostenibilitá: il modello SLoT, FrancoAngeli, Milano.

Harvey, D. (2004). Espacios de la esperanza. (Ed. Castellano) Akal, Madrid.

Howitt, R. (1993). "A World in a Grain of Sand: Towards a Reconceptualization of Geographical Scale", Australian Geographer, 24, 1, 33-44.

Magnaghi, A. (2000). Il progetto locale. Bollati Boringhieri, Milano.

Marsden T., Murdoch, J. Lowe, P., Munton, R. y Flynn, A. (1993). Constructing the countryside. UCL Press, London.

Merrifield, A. y Swyngedow, E. (1997). The urbanization of. injustice. New York University Press. 
Molina, M. (2004a). "El desarrollo local: problemática y estrategias futuras" en Territorio y Desarrollo Local, II Época, n.1. Revista Trimestral del Departamento de Presidencia y Relaciones Institucionales del Gobierno de Aragón.

Molina, M. (2004b). "Mycologie et nouvelles technologies: Un modèle de gestion durable". En: Technologies de Information et Développement Économique Local. Insprom. Publisud. París. 2004.

Moyano Estrada, E. y Paniagua Mazorra, A.: "Medio ambiente, desarrollo sostenible y escalas de sustentabilidad" en Reis: Revista española de investigaciones sociológicas № 83, 1998: 151-175.

Pasqui,G. (2006). "Le politiche di sviluppo regionale in Italia.Un seminario" en Territorio n.38 p. 108-110.

Perelli, C. Pinna, P. Sistu, G (2010). "Mining Heritage, Local Development and Identity: The Case of Sardinia" en Conlin,M. y Jolliffe,L. Mining Heritage and Tourism A Global Synthesis, Routledge Oxford.

Santos, M. (1994). "O retorno do territorio", en Santos, M.-De Souza,M.A.-Silveira, M.L Territorio. Globalização e Fragmentação. HUCITEC, Sao Paulo.
Santos, M. (1996). De la totalidad al lugar. Ed española Oikos Tau, Barcelona.

Sassen, S. (1991). The global city. New York, London, Tokyo. Princeton University.

Sassen, S. (2005). Contrageografias de la globalización. Género y ciudadanía en los circuitos transfronterizos. Copyleft Traficantes de sueños, Madrid.

Sassu, A. eds. (2004). Saperi locali in Sardegna: tradizione e innovazione nell'attività económica. CUEC, Cagliari.

Storper, M. y Venables, A. (2004). "Buzz: faceto-face contact and the urban economy". Journal of Economic Geography. August 2004; 4, 4, pp. 351-370.

Swyngedouw, E. (2000). Authoritarian governance, power and the politics of rescaling. En Environment and Planning: Society and Space, vol 18.

Villarino Pérez, M. y Armas Diéguez, P. (2000). "La aldea global y el trabajo a domicilio en Galicia: "coser y cantar" en Garcia Ramon, M. D; Baylina Ferré, M (eds.) El nuevo papel de las mujeres en el desarrollo rural. Vilassar de Mar: OikosTau, pp. 245-268. 